\title{
Conditional emergence of classical domain and branching of quantum histories
}

\author{
Alexei V. Tkachenko ${ }^{*}$ \\ Center for Functional Nanomaterials, Brookhaven National Laboratory, Upton New York 11973, USA
}

(Received 16 September 2019; accepted 30 September 2020; published 5 November 2020)

\begin{abstract}
We outline the minimalistic measurement scheme (MMS) compatible with regular unitary evolution of a closed quantum system. Within this approach, a part of the system becomes informationally isolated (restricted) which leads to a natural emergence of the classical domain. This measurement scenario is a simpler alternative to environment-induced decoherence that reconciles unitarity of quantum mechanics $(\mathrm{QM})$ with irreversible measurement process. In its basic version, MMS involves two ancilla qubits, $A$ and $X$, entangled with each other and with the system $S$. Informational or thermodynamic cost of measurement is represented by the $X$ qubit being isolated, i.e., becoming unavailable for future interactions with the rest of the system. Conditional upon this isolation, the $A$ qubit, which plays the role of an apparatus, becomes classical and records the outcome of the measurement. Classicality is therefore both an emergent and reversible property, since breaking informational isolation of the $X$ qubit would bring the apparatus back to the quantum realm. The MMS procedure may be used to perform von Neumann-style projective measurements or generalized ones, which corresponds to a positive-operator-valued measure (POVM). By repeating the same generalized measurement multiple times with different $A$ and $X$ qubits, one asymptotically approaches the wave-function collapse in the basis determined by the premeasurement process. We present a simple result for the total information extracted after $N$ such weak measurements. Building upon MMS, we propose a construction that maps a history of a quantum system onto a set of $A$ qubits. It resembles the consistent histories $(\mathrm{CH})$ formulation of quantum mechanics but is distinct from it and is built entirely within the conventional QM. In particular, the consistency postulate of the $\mathrm{CH}$ formalism is not automatically satisfied but is rather an emerging property. Namely, each measurement event corresponds to the branching of mutually exclusive classical realities whose probabilities are additive. In a general case, however, the superposition between different histories is determined by the history density matrix.
\end{abstract}

DOI: 10.1103/PhysRevResearch.2.043188

\section{INTRODUCTION}

As it approaches its centennial, quantum mechanics (QM) is still commonly perceived as a counterintuitive and mysterious field of physics. Most of its postulates and the overall mathematical structure, although exotic at the time of their development, are relatively straightforward. However, there is one important element of QM that remains puzzling and controversial and is responsible for many of its paradoxical aspects: the measurement problem [1-6]. In QM, the state of a system is described as a vector in Hilbert space, called a wave function $|\Psi\rangle$, and its time evolution is given by a linear unitary operator: $|\Psi\rangle \rightarrow \hat{T}|\Psi\rangle$. This evolution can be calculated, e.g., with the help of the Schrödinger equation, Heisenberg's matrix mechanics, or Dirac-Feynman path integrals. According to the conventional Copenhagen formulation of QM, at the moment of measurement, the regular unitary evolution stops working and the wave function "collapses," i.e., the system

\footnotetext{
*oleksiyt@bnl.gov

Published by the American Physical Society under the terms of the Creative Commons Attribution 4.0 International license. Further distribution of this work must maintain attribution to the author(s) and the published article's title, journal citation, and DOI.
}

ends up in one of the eigenstates of the corresponding operator. This prescription is practical but conceptually problematic since the observer is assumed to live in a classical world rather than being described by QM itself.

There is a long history of research into the topic of quantum measurement, starting with John von Neumann's scheme proposed in the early days of QM [1]. He demonstrated how a measuring apparatus operating according to the laws of QM can be inserted between the measured system and the observer. He also formalized the wave-function collapse process in the form of the projection postulate. According to it, the wave function, at the time of measurement, changes as $|\Phi\rangle \rightarrow \hat{P}_{k}|\Phi\rangle / \sqrt{p_{k}}$ where $\hat{P}_{k}=|k\rangle\langle k|$ is the projection operator associated with the observed eigenstate $|k\rangle$, and $p_{k}=\left\langle\Phi\left|\hat{P}_{k}\right| \Phi\right\rangle$ is the probability of that particular outcome. von Neumann's theory does not resolve the quantum measurement problem but rather restates mathematically the Copenhagen-style prescription of wave-function collapse. In particular, von Neumann argues that an interface between the quantum and classical worlds is ultimately unavoidable since any measurement has to be eventually perceived by an observer who lives in a classical reality. Attempts to resolve the quantum-classical conundrum included an exotic attempt by Bohm to couple wave functions to real particle dynamics [3], as well as a multiworld interpretation by Everett [4,5]. Since the original paper of Everett, this approach has evolved 
into a sophisticated though still controversial alternative to the simplistic Copenhagen interpretation of QM [7].

An important breakthrough in understanding the nature of quantum measurements came with the so-called environmentinduced decoherence (EID) program introduced in the works of Zurek, Gell-Mann, Hartle, and others [8-11]. It is based on the observation that any practical measurement device interacts with an environment. The decoherence associated with this interaction explains, at least partially, the branching of the system between different classical realities that represent different outcomes of the measurement. However, in the studied examples with explicit models of the environment, the perfect measurement is only achieved in the thermodynamic limit [9]. So, even the simplest quantum system could be described self-consistently only as a part of an infinitely large one. As a result, within the decoherence program, the fundamental problem of quantum measurement becomes dependent on our ability to understand statistical properties of a complex system. Partially because of this, its generality and limits of applicability are not fully established.

In this paper, we propose a simple, self-consistent description of quantum reality, which is built strictly within the conventional QM, yet without the introduction of the wave-function collapse or projection postulate. We also do not include traditional EID, primarily for the sake of greater simplicity. Based on quantum information theory we argue that a measurement process unavoidably requires a loss of information. Within our approach, the minimalistic measurement scheme (MMS), this informational sacrifice for each measurement is represented by a single qubit that becomes informationally isolated, i.e., unavailable for any future interactions. Conditional upon its isolation, the classical domain naturally emerges within a quantum system. In effect, the MMS is a greatly simplified version of the decoherence approach which captures the emergence of classically but does not address other delicate aspects of the measurement problem, such as so-called "superselection." Following a brief review of von Neumann theory and the decoherence program in Sec. II, we describe the MMS in Sec. III and demonstrate how it can be used to make von Neumann-style projective measurements, as well as generalized quantum measurements, described as positive-operator-valued measures (POVMs). Building upon this approach, in Sec. IV we present a construction that maps a history of a quantum system onto a set of ancilla qubits that are consequently subjected to MMS. Our approach resembles but is not equivalent to the consistent histories $(\mathrm{CH})$ formalism. While $\mathrm{CH}$ is built upon a set of postulates of its own, our construction is done within the traditional framework of QM, with measurements implemented through MMS. In particular, one of the central elements of $\mathrm{CH}$, the consistency postulate, is not automatically satisfied. Instead, it is an emerging property, conditional upon informational isolation of a part of the system, as any classical information within the MMS. Within the original $\mathrm{CH}$ approach, a quantum history is defined as a chain of projections and unitary evolution operators. In our construction, we generalize this definition by replacing projectors with Kraus operators associated with POVM-like weak measurements.

\section{VON NEUMANN MEASUREMENT THEORY AND DECOHERENCE PROGRAM}

To model the measuring process, von Neumann considered a combination of two quantum subsystems: the system $S$, and the apparatus $A$ [1]. During the first step, which is called premeasurement, a quantum entanglement between these two subsystems is achieved. Namely, if $S$ is in a quantum state $\sum_{k} c_{k}|k\rangle$, and the apparatus is originally in state $|0\rangle_{A}$, the premeasurement is the following unitary transformation:

$$
\left(\sum_{k} c_{k}|k\rangle\right)|0\rangle_{A} \rightarrow|\Psi\rangle=\sum_{k} c_{k}|k\rangle|k\rangle_{A} .
$$

Here $|k\rangle$ and $|k\rangle_{A}$ are states of $S$ and $A$, respectively. Following the premeasurement, the wave-function collapse is described as a nonunitary transformation of the density operator, $\hat{\rho}=|\Psi\rangle\langle\Psi|:$

$$
\hat{\rho} \rightarrow \sum_{k} \hat{P}_{k} \hat{\rho} \hat{P}_{k}
$$

Here $\hat{P}_{k}=|k\rangle_{A}\left\langle\left. k\right|_{A}\right.$ are projection operators of the apparatus subsystem. If both the premeasurement (1), and the nonunitary projection process (2), are performed in the same basis of the apparatus states, $|k\rangle_{A}$, they would transform the density operator of the combined system into the diagonal form:

$$
\hat{\rho}=\sum_{k}\left|c_{k}\right|^{2}|k\rangle|k\rangle_{A}\left\langle\left. k\right|_{A}\langle k|\right.
$$

Here $\left|c_{k}\right|^{2}$ are probabilities of different results, and since all the off-diagonal terms are zeros, there is no interference between those outcomes, i.e., the superposition principle of the classical probability theory is recovered.

The nonunitary projection process is certainly inconsistent with conventional quantum dynamics. A reasonable justification for it was given much later, within the decoherence program [8-11]. Let us imagine that an environment, subsystem $E$, is coupled to the apparatus in such a way that the states $|k\rangle_{A}$ of the latter are preserved in time, but the evolution of the environment depends on that state. As a result, following the premeasurement, Eq. (1), the composite $S+A+E$ system will evolve after some time into a new state:

$$
|\Psi\rangle|0\rangle_{E}=\left(\sum_{k} c_{k}|k\rangle|k\rangle_{A}\right)|0\rangle_{E} \rightarrow \sum_{k} c_{k}|k\rangle|k\rangle_{A}|k\rangle_{E} .
$$

The density operator of system $S+A$ is obtained by taking a partial trace of the overall $S+A+E$ density operator with respect to environment variables, $\hat{\rho}_{s a}=\operatorname{Tr}_{e} \hat{\rho}_{s a e}$ At this stage, one would recover the von Neumann nonunitary measurement process, Eq. (2), if the eventual states of environment that correspond to different indexes $k$ are mutually orthogonal: $\left\langle k \mid k^{\prime}\right\rangle_{E}=\delta_{k k^{\prime}}$. This orthogonality condition can indeed be proven directly for certain explicit models of the environment, but it is typically achieved only in the thermodynamic limit [8]. 


\section{MINIMALISTIC MEASUREMENT SCHEME}

\section{A. Conditional emergence of classical domain}

An important insight into the nature of a quantum measurement is given by quantum information theory [1,12-14]. Quantum information entropy, also introduced by von Neumann, $S=-\operatorname{Tr}\left(\hat{\rho} \log _{2} \hat{\rho}\right)$ is zero for the system in a pure quantum state and is conserved by unitary evolution. Therefore, if the initial quantum state of an isolated system is known, its information entropy is $S=0$. If one now performs a new measurement on the same system and records its result with a classical bit, the information entropy associated with that bit is $\Delta S=-p \log _{2} p-(1-p) \log _{2}(1-p)$, where $p$ is the probability of it being in state 1 . After an observer reads the bit, this information is extracted, and the measured system once again returns to a pure state with $S=0$. We come to a seemingly paradoxical conclusion that the new information is extracted from nowhere. The nonunitary von Neumann process and the decoherence program both provide a partial resolution to this paradox. In both cases, the information entropy of the system is being increased during the measurement. This allows one to reduce $S$ back to zero once the result of the measurement is read and to record the new information about the system. In other words, either process is needed to erase some information.

Here we introduce the minimalistic measurement scheme (MMS) in which this sacrifice of information is represented by a single qubit which becomes unavailable for any future interactions. Consider a toy model consisting of three qubits: system $S$ and two ancilla qubits $A$ and $X$. The $A$ qubit plays the role of an apparatus and eventually will record the measurement outcome, while the $X$ qubit will be discarded. Prior to the measurement, $S$ is in an unknown state $|\psi\rangle=c_{1}|1\rangle+$ $c_{0}|0\rangle$ while both $A$ and $X$ are prepared in their respective "zero" states, $|0\rangle_{A}$ and $|0\rangle_{X}$. The measurement protocol is as follows: first, we perform the premeasurement with the help of quantum CNOT gate acting on $S$ and $A$, and then execute the same operation on $A$ and $X$. CNOT (or controlled NOT) is one of the most common gates in quantum logic [15]. When acting on the system and apparatus with our initial conditions, it would results in von Neumann-style entanglement between them: $c_{1}|1\rangle|1\rangle_{A}+c_{0}|0\rangle|0\rangle_{A}$. Following this two-step process, all three qubits would become entangled:

$$
|\psi\rangle|0\rangle_{A}|0\rangle_{X} \rightarrow c_{1}|1\rangle|1\rangle_{A}|1\rangle_{X}+c_{0}|0\rangle|0\rangle_{A}|0\rangle_{X}
$$

This is a unitary process fully consistent with QM. Following it, we assume the $X$ qubit to become unavailable for any future interactions. Mathematically, this would imply that, instead of considering the full $S+A+X$ system, we should calculate the density operator of the reduced $S A$ system by taking the partial trace with respect to the $X$ qubit:

$$
\hat{\rho}_{s a}=\operatorname{Tr}_{X} \hat{\rho}_{s a x}=\left|c_{1}\right|^{2}|1\rangle|1\rangle_{A}\left\langle\left. 1\right|_{A}\left\langle\left. 1|+| c_{0}\right|^{2} \mid 0\right\rangle \mid 0\right\rangle_{A}\left\langle\left. 0\right|_{A}\langle 0| .\right.
$$

This result is formally equivalent to the wave-function collapse as given by von Neumann's nonunitary projection process, Eq. (2). The overall measurement process is schematically shown in Fig. 1.

The classical domain within our toy model emerges as a direct consequence of ignoring information about the $X$

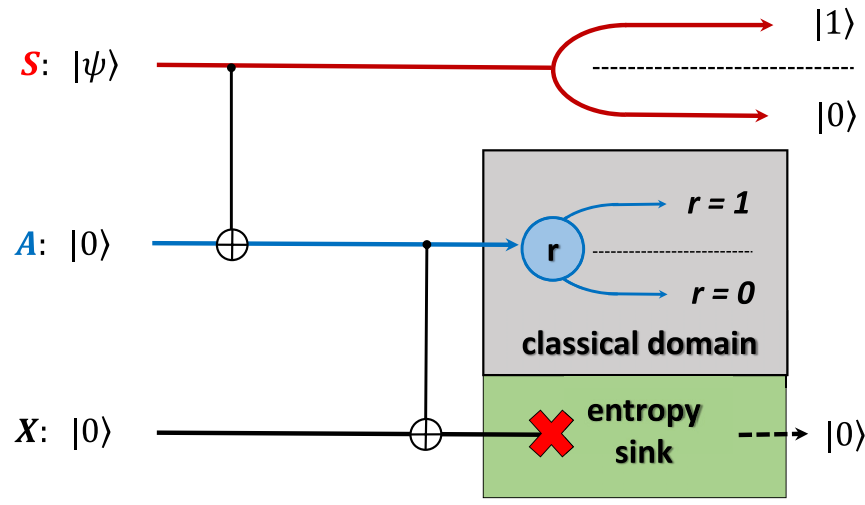

FIG. 1. Schematic representation of the minimalistic measurement scheme (MMS).

qubit. There are several ways in which this process can be interpreted and/or implemented. First, one can employ an additional device that acts as the entropy sink. Its role is to supply a new $X$ qubit in a pure quantum state $|0\rangle$, in exchange for the current one. Upon this exchange, we recover our original $S+A+X$ system, but the apparatus now becomes a classical bit that has recorded the result of the measurement, $r$, while system $S$ ends up in a pure quantum state $|r\rangle$ for each of the outcomes. Information entropy associated with the measurement, $\Delta S=-\sum_{r} p_{r} \log _{2} p_{r}$, is equal to the one adsorbed by entropy sink, together with the $X$ qubit. If we consider $S+A+X$ as a single quantum system, its entropy would remain 0 . This means that the positive entropy $2 \Delta S$ of the two separated qubits is equal and opposite to the entropy associated with the classical correlation and quantum entanglement between them (each contributing $-\Delta S$ to the overall quantum information entropy) [12-14,16,17]. This negative mutual entropy is lost due to separation. Furthermore, if the design of the entropy sink is such that any information about the $X$ qubit is getting effectively erased due to the underlying ergodicity $[18,19]$, the ultimate entropy cost of a single-bit measurement is given by the maximum possible value of $\Delta S$, i.e., 1. This result is consistent with Landauer's principle that sets the lower bound for the energy cost needed for irreversible single-bit operation at finite temperature $T$ : $E \min =k_{B} T \ln 2[20,21]$.

An alternative to the introduction of the entropy sink is a notion that classicality is a property conditional upon certain information, i.e., the $X$ qubit, remaining restricted. Note that the choice between $A$ and $X$ is arbitrary: each of them can be used to record the result, while the other can be the "lost" qubit. Thus, one can imagine two independent observers, Alice and Bob, to have access to $A$ and $X$ qubits, respectively. This way, each of them would have a classical record of the same measurement, as long as they are not allowed to communicate with each other. If this condition is ever broken, i.e., any information is exchanged between them, the nonclassical correlations, such as the violation of Bell's inequality, would become possible [22,23]. This way, the classicality of the $A$ qubit would be effectively "erased."

The above procedure, while being relatively trivial, does provide a resolution to some of the issues related to the quantum measurement problem. In particular, the combined 
$S+A+X$ system follows a regular unitary evolution at any time. The classicality of the apparatus is an emerging property, subject to the condition of informational isolation of the $X$ qubit after the measurement. The entanglement entropy between $A$ and $X$ qubits constitute the informational or thermodynamic cost of the measurement. One aspect that this simplistic approach does not address is the so-called "superselection," i.e., it does not differentiate between "observable" and "nonobservable" quantum states. Under the decoherence program, it is suggested that the basis of so-called "pointer" states is chosen by means of environment-induced superselection or einselection. It implies that the form of coupling between the apparatus and the environment predetermines the observable that is being measured.

\section{B. Generalized measurements within the minimalistic measurement scheme}

Now we consider a variation of the above minimalistic scheme in which the basis of the apparatus states during the two stages of the measurement process are not the same. Specifically, following the premeasurement, $\operatorname{CNOT}(S, A)$, and prior to the interaction with the $X$ qubit, we can apply a unitary rotation operator to the apparatus qubit:

$$
\hat{R}_{A}(\theta, \phi)=\left[\begin{array}{cc}
\cos \theta & e^{i \phi} \sin \theta \\
-e^{-i \phi} \sin \theta & \cos \theta
\end{array}\right]_{A} .
$$

After that, the $A$ and $X$ qubits are entangled by means of $\operatorname{CNOT}(A, X)$ quantum gate. As a result, our original state is transformed by three consecutive unitary operations, as shown in Fig. 2(a):

$$
|\Psi\rangle_{\theta, \phi}=\operatorname{CNOT}(A, X) \times \hat{R}_{A}(\theta, \phi) \times \operatorname{CNOT}(S, A)|\psi\rangle|0\rangle_{A}|0\rangle_{X} .
$$

As before, the $X$ qubit is "discarded" and we can calculate the resulting density operator of the $S+A$ system by taking the partial trace over the $X$ subsystem:

$$
\hat{\rho}_{s a}=\operatorname{Tr}_{X}|\Psi\rangle_{\theta, \phi}\left\langle\left.\Psi\right|_{\theta, \phi}=\sum_{r=0,1} \mid r\right\rangle_{A}\left|S_{r}\right\rangle\left\langle S_{r}\right|\left\langle\left. r\right|_{A} .\right.
$$

Here $|1\rangle_{A}$ and $|0\rangle_{A}$ form the basis of the apparatus states upon rotation, Eq. (7). Just like in the previous case, the apparatus becomes completely classical: there is no quantum interference between $|1\rangle_{A}$ and $|0\rangle_{A}$. This corresponds to the two alternative results of measurement, $r=1$ or 0 . The corresponding system states are

$$
\left|S_{r}\right\rangle=\hat{M}_{r}(\theta, \phi)|\psi\rangle .
$$

Here $\hat{M}_{r}(\theta, \phi)$ are known as Kraus operator [24] for outcomes $r=1,0$, respectively:

$$
\begin{aligned}
\hat{M}_{r}(\theta, \phi) & =\sum_{k=1,0} R_{r k}^{*}(\theta, \phi) \hat{P}_{k} \\
& = \begin{cases}\hat{P}_{1} \cos \theta+\hat{P}_{0} e^{-i \phi} \sin \theta, & r=1 \\
-\hat{P}_{1} e^{i \phi} \sin \theta+\hat{P}_{0} \cos \theta, & r=0 .\end{cases}
\end{aligned}
$$

Here $\hat{R}^{*}(\theta, \phi)$ is the complex-conjugate of the rotation matrix $\hat{R}(\theta, \phi)$ given by Eq. (7). The two state vectors $\left|S_{1}\right\rangle$ and $\left|S_{0}\right\rangle$ are in a general case not orthogonal, and not yet normalized. The probabilities of the corresponding outcomes can be found

\section{(a) Generalized measurement}

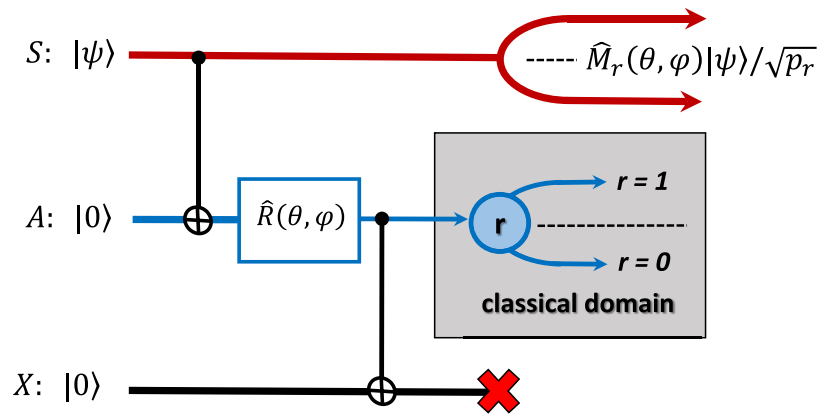

(b) Premeasurement "eraser"

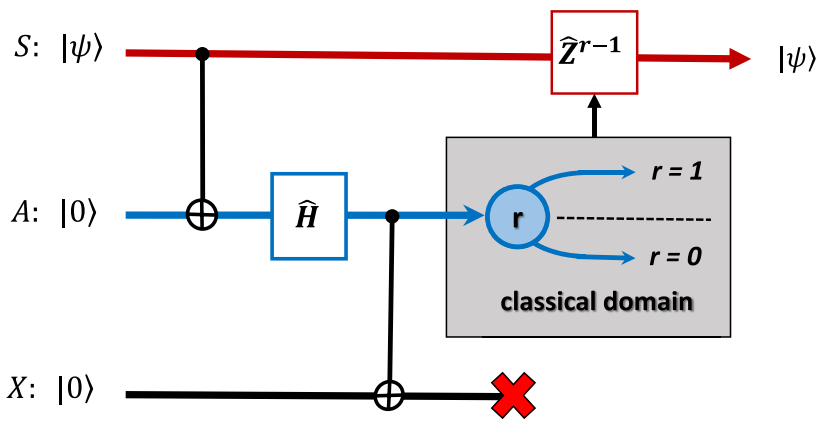

FIG. 2. (a) Generalized quantum measurement with the MMS. (b) Erasing the premeasurement event. $\hat{H}=\hat{R}(\pi / 4,0)$ is a Hadamard quantum gate. The outcome of the measurement $r=1 \mathrm{oc}-$ curs with $50 \%$ probability and indicates that the system has returned to its original state, with any effect of the premeasurement reverted. For $r=0$, the original state can be recovered with the help of a Pauli $Z$ gate.

in a standard QM manner:

$$
p_{r}=\left\langle S_{r} \mid S_{r}\right\rangle .
$$

Importantly, von Neumann's information entropy associated with the density operator $\hat{\rho}_{s a}$ in Eq. (9), coincides with the Shannon's entropy that can be calculated based on these probabilities:

$$
S=-\operatorname{Tr}\left(\hat{\rho}_{s a} \log _{2} \hat{\rho}_{s a}\right)=-\sum_{r=0,1} p_{r} \log _{2} p_{r} .
$$

This means that the density operator represents a so-called "ignorance interpretable" mixture of pure states. In other words, once the result of the measurement is known, the system ends up in pure quantum state, $\left|S_{r}\right\rangle / \sqrt{p_{r}}$. However, in a general case, the specific states that correspond to each outcome are not predetermined by the measurement. Indeed, $\hat{M}_{r}(\theta, \phi)$ in Eq. (11) does not have the conventional form of a projection operator $\hat{P}_{k}=|k\rangle\langle k|$, so the final states will depend on the state of the system prior to the measurement, $|\phi\rangle$. In particular, one of the most important properties of the projection operator, $\hat{P}_{k} \hat{P}_{k^{\prime}}=\delta_{k k^{\prime}} \hat{P}_{k}$, is violated for $\hat{M}_{r}(\theta, \phi)$. This is an example of the generalized quantum measurement, associated with the positive-operator-valued measure (POVM) [24-26]. One of their signatures is that they are in 
general nonrepeatable. When two identical measurements are performed with the same system but with two different apparatuses, they are not guaranteed to give the same result. This is a spectacular violation of the projection postulate, although the latter is still valid for the combined $S+A$ system.

An important difference from the regular von Neumann projection is that the system, unlike the apparatus, is not becoming classical immediately after the measurement but ends up in a new coherent quantum state. Furthermore, following the premeasurement process, the $A$ qubit can in principle be stored under decoherence-free conditions for as much time as feasible. The measurement itself can be done later, in any basis of one's choice. One can even completely "erase" the fact of the premeasurement $[27,28]$. To do so, the rotation operator must be given by Hadamard quantum gate, $\hat{H}=\hat{R}(\pi / 4,0)$. If now the result of the measurement is 1 , it would mean that any effect of the premeasurement on the system has been successfully reverted. This is because $\hat{P}_{1}(\pi / 4,0)=\hat{I} / \sqrt{2}$. The probability of that outcome is $1 / 2$. Its alternative, $r=0$, also corresponds to a unitary transformation, Pauli $Z$ gate, applied to the system: $\hat{P}_{0}(\pi / 4,0)=\left(\hat{P}_{0}-\hat{P}_{1}\right) / \sqrt{2}=\hat{Z} / \sqrt{2}$. If the result of the measurement is 0 , the system can still be returned back to its original state by the same transformation, as shown in Fig. 2(b).

\section{Asymptotic collapse of wave function}

In the above example, we returned the system back to its original state, and no information was extracted. On the other hand, the opposite limit when the measurement of the apparatus state is done in its original basis, as shown in Fig. 1, corresponds to a traditional projection measurement. The quantum state of the system after the measurement is completely determined by the result, but its prior state is destroyed. The generalized measurement shown in Fig. 2(a) is an intermediate case. Only a fraction of the information about the state of the system after the measurement is extracted, and the system may still, in principle, be returned to its original state $|\phi\rangle$. This is an example of a weak quantum measurement [29,30].

By repeating the same weak measurement for multiple times with different pairs of $A$ and $X$ qubits, one would achieve an asymptotic wave-function collapse. It is in principle reversible [29,30], although with exponentially diminishing probability. Below we explicitly calculate the amount of information extracted after $N$ such weak measurements are made. Note that the number $l$ of $A$ qubits that end up in state $r=1$ completely determine the overall Kraus operator associated with these $N$ measurements:

$$
\hat{M}_{l}=\hat{P}_{1} \cos ^{l} \theta\left(-e^{i \phi} \sin \theta\right)^{N-l}+\hat{P}_{0} \cos ^{N-l} \theta\left(e^{-i \phi} \sin \theta\right)^{l} .
$$

The probability of a given value $l$ can be found as

$$
p_{l}=\left(\begin{array}{c}
N \\
l
\end{array}\right)\left[\left|c_{1}\right|^{2} q^{2 l}\left(1-q^{2}\right)^{N-l}+\left|c_{0}\right|^{2} q^{2(N-l)}\left(1-q^{2}\right)^{l}\right] .
$$

Here $q=\cos \theta$. One can see that this result is a linear combination of two binomial distributions, $B_{l}\left(q^{2}, N\right)=\left(\begin{array}{c}N \\ l\end{array}\right) q^{2 l}(1-$ $\left.q^{2}\right)^{N-l}$ and $B_{l}\left(1-q^{2}, N\right)$, with coefficients given by probabilities of the system to be in state $|1\rangle$ and $|0\rangle$, respectively. In

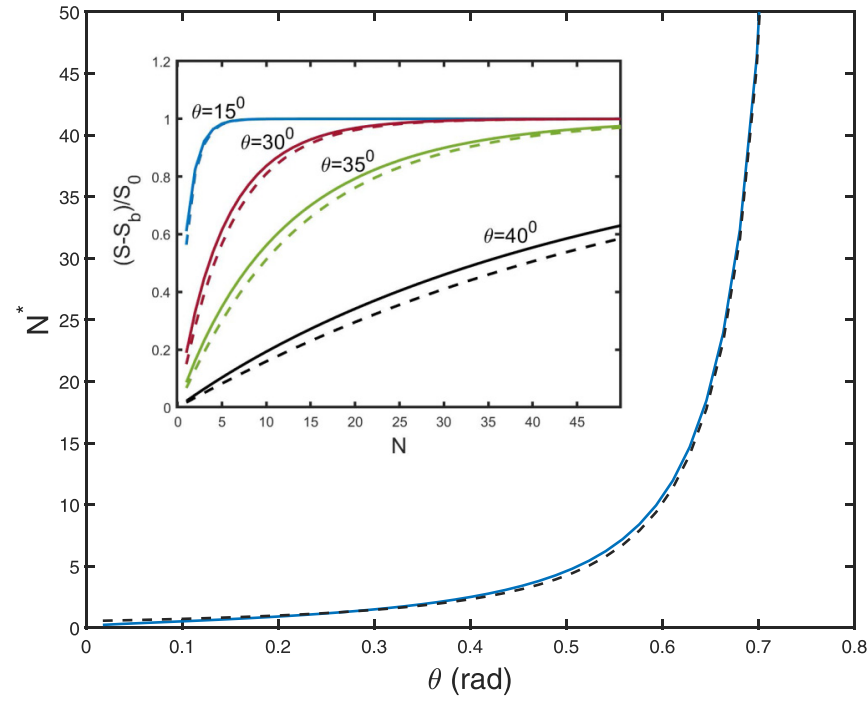

FIG. 3. Gradual "collapse" of the wave function after multiple weak measurements. Characteristic number of measurements needed for information extraction, $N^{*}$ is plotted vs rotation angle $\theta$ (solid line). Dashed line shows asymptotic result, Eq. (18). Inset shows amount of information extracted vs number of measurements, $N$, for different values of $\theta$ (solid lines correspond to $\left|c_{0}\right|^{2}=\left|c_{1}\right|^{2}=1 / 2$, dashed lines correspond to $\left|c_{0}\right|^{2}=0.1$ ).

the limit of large $N$ these distributions are strongly peaked at values $l_{1}=N q^{2}$ and $l_{0}=N\left(1-q^{2}\right)$, which is an indication of an asymptotic wave-function collapse in the basis determined by the premeasurement. In this limit, the information entropy of the overall distribution, $S=-\sum_{l} p_{l} \log _{2} p_{l}$, is given by

$$
S \rightarrow S_{b}\left(q^{2}, N\right)-\sum_{k=1,0}\left|c_{k}\right|^{2} \log _{2}\left(\left|c_{k}\right|^{2}\right) .
$$

Here the first contribution is the entropy of the binomial distribution,

$$
S_{b}\left(q^{2}, N\right)=-\sum_{l} B_{l}\left(q^{2}, N\right) \log _{2} B_{l}\left(q^{2}, N\right),
$$

and the second one is the total information entropy extracted from the system, $S_{0}$. In the insert of Fig. 3 we show how much of this information is extracted, as a function of $N$, for various values of the basis rotation angle $\theta$. One can see that the quantity $\left[S-S_{b}\left(q^{2}, N\right)\right] / S_{0}$ exponentially approaches 1 , essentially independently of the original state of the system, i.e.,

$$
S-S_{b}\left(q^{2}, N\right)=S_{0}\left[1-\exp \left(-N / N^{*}\right)\right] .
$$

Naturally, the parameter $N^{*}$ diverges in the vicinity of $\theta=\pi / 4$ since no information can be extracted at that angle. One can determine the asymptotic behavior of $N^{*}$ for small values of $x=\theta-\pi / 4 \approx 1 / 2-q^{2}$. To do this, we consider the case when the two states of the system have the same probability (i.e., $S_{0}=1$ ) and calculate the leading contribution in $x$ to the amount of entropy extracted after $N$ measurements: $S-S_{b}\left(q^{2}, N\right) \approx 2 x^{2} N / \ln 2$. This leads to the following result for the characteristic number of the required 
weak measurements:

$$
N^{*} \approx \frac{\ln 2}{2(\theta-\pi / 4)^{2}} .
$$

Remarkably, this asymptotic formula gives a near perfect fit across the whole range of $\theta$, as shown in Fig. 3 .

\section{BRANCHING OF QUANTUM HISTORIES}

Now we consider a more general case: a system consisting of $d$ qubits undergoes a unitary evolution. At certain moments of time it gets sequentially entangled with $n$ different qubits: $A_{1}, A_{2}, \ldots, A_{n}$, representing $n$ distinct premeasurement events. In a general case, these events are time ordered, but some of them may occur at the same time, or the order within a certain subgroup of them may be not important. As discussed above, the $A$ qubit can in principle be stored for some time before the actual measurement is done. The resulting state of the system with all $n$ such qubits can be expressed as

$$
|\Psi\rangle=\sum_{\alpha}\left|\alpha_{1}\right\rangle_{1}\left|\alpha_{2}\right\rangle_{2} \cdots\left|\alpha_{n}\right\rangle_{n} \hat{C}_{\alpha}\left|\psi_{0}\right\rangle .
$$

Here $\left|\alpha_{l}\right\rangle_{l}$ represents the state of the ancilla qubit $A_{l}\left(\alpha_{l}=\right.$ 0 or 1$)$, and $\alpha=\left(\alpha_{1}, \alpha_{2}, \ldots, \alpha_{n}\right)$ is a vector that represents the combined state of all $n A$ qubits. $\hat{C}_{\alpha}$ is known as a chain operator, which is a series of projectors $\hat{P}_{\alpha_{l}}^{(l)}$ and time evolution operators $\hat{T}\left(t_{l-1} \rightarrow t_{l}\right)$ :

$$
\hat{C}_{\alpha}=\prod_{l=1}^{n} \hat{P}_{\alpha_{l}}^{(l)} \hat{T}\left(t_{l-1} \rightarrow t_{l}\right) .
$$

One may notice that our construction, Eqs. (19) and (20), strongly resembles the consistent histories $(\mathrm{CH})$ approach to QM [10,31-34]. In its language, each vector $\alpha$ would represent a specific history of the quantum system. Despite this similarity, there are important conceptual differences between the present approach and the $\mathrm{CH}$ formalism. First, our construction is done entirely within the framework of conventional QM (though without explicit wave-function collapse or projective postulate), while $\mathrm{CH}$ is built on its own set of postulates. Second, the fundamental space within $\mathrm{CH}$ is a tensor product of Hilbert spaces of the original system at different times. In contrast, we simply expanded the system's Hilbert space at a given time by combining it with that of $n \mathrm{~A}$ qubits.

The overall density operator of the combined system, $\hat{\rho}=$ $|\Psi\rangle\langle\Psi|=| \alpha\rangle \hat{C}_{\alpha} \hat{\rho}_{0} \hat{C}_{\alpha^{\prime}}^{*}\left\langle\alpha^{\prime}\right|$ can be expressed as a $2^{n} \times 2^{n}$ matrix in which each element is itself an operator in the system's Hilbert space: $\hat{\rho}_{\alpha \alpha^{\prime}}=\left\langle\alpha|\hat{\rho}| \alpha^{\prime}\right\rangle=\hat{C}_{\alpha} \hat{\rho}_{0} \hat{C}_{\alpha^{\prime}}^{*}$. By taking a partial trace over system's final states, we obtain the reduced density operator that can be called a history density matrix:

$$
D_{\alpha \alpha^{\prime}}=\operatorname{Tr}_{S}\left(\hat{C}_{\alpha} \hat{\rho}_{0} \hat{C}_{\alpha^{\prime}}^{*}\right) \text {. }
$$

Furthermore, our assumption that the system was prepared in a particular pure state $\left|\psi_{0}\right\rangle$ is in fact redundant: if needed, the preparation can be implemented as a set of initial measurements. Under the assumption that only $A$ qubits are available for measurements, we should take a partial trace over all plausible initial states of the system, which amount to setting its a priori density operator to $\hat{\rho}_{0}=2^{-d} \hat{I}$. One of the central postulates of $\mathrm{CH}$, known as the consistency condition, would require mutual orthogonality of different histories, i.e., $D_{\alpha \alpha^{\prime}}=$ 0 for $\alpha \neq \alpha^{\prime}$. However, this condition is not automatically satisfied within our construction, reflecting the constraints of the standard QM to which we adhere. The history density matrix, Eq. (21), is in fact a direct analog of the decoherence functional used by Hartle and Gell-Mann for describing the effect of coarse graining on quantum histories in continuous space-time [10,34].

We can now employ MMS described in the previous section: each $A$ qubit is first subjected to unitary transformation $\hat{R}^{(l)}$, followed by entanglement with the respective $X$ qubit. If all $n$ of those become informationally isolated or discarded, the whole history vector $\beta=\left(\beta_{1}, \ldots, \beta_{n}\right)$ is measured. Following this, all the off-diagonal elements of the new history density matrix $\hat{\mathbf{D}}$ will be set to zero: $\mathbf{D}_{\beta \beta^{\prime}}=\delta_{\beta \beta^{\prime}} \operatorname{Tr}\left(\hat{\mathbf{C}}_{\beta} \hat{\rho}_{0} \hat{\mathbf{C}}_{\beta}^{*}\right)$, which coincides with the consistency postulate of $\mathrm{CH}$ formalism. Note that the chain operator has been redefined and will now be called the generalized history operator:

$$
\hat{\mathbf{C}}_{\beta}=\prod_{l=1}^{n} \hat{M}_{\beta_{l}}^{(l)} \hat{T}\left(t_{l-1} \rightarrow t_{l}\right) .
$$

Compared to the traditional definition of $\hat{C}_{\alpha}$, Eq. (20), it incorporates the possibility of weak or generalized measurements. As one can see, the projectors $\hat{P}$ have been replaced with Kraus operators $\hat{M}$ which correspond to POVM rather than regular projective measurements. As has been shown in the previous section, these operators are related to the rotations of the corresponding $A$ qubits:

$$
\hat{M}_{\beta_{l}}^{(l)}=\sum_{k=0,1} \hat{R}_{\beta_{l} k}^{(l) *} \hat{P}_{k}^{(l)}
$$

If only a subset of the $A$ qubits has been subjected to MMS, a history can be represented as a logical conjunction $\beta \wedge \gamma$ of $m$ measured and $n-m$ unmeasured binary variables: $\beta=$ $\left(\beta_{1}, \ldots, \beta_{m}\right)$ and $\gamma=\left(\alpha_{m}+1, \ldots, \alpha_{n}\right)$, respectively (without loss of generality, we can re-order the $A$ qubits so that the first $m$ are measured). The overall construction is illustrated in Fig. 4. After the measurement, the system branches onto $2^{m}$ distinct classical realities parametrized by vector $\beta$. The postmeasurement history density matrix will have the following form:

$$
\mathbf{D}_{(\beta \wedge \gamma)\left(\beta^{\prime} \wedge \gamma^{\prime}\right)}=\delta_{\beta \beta^{\prime}} \mathbf{D}_{\gamma \gamma^{\prime}}^{(\beta)}=\delta_{\beta \beta^{\prime}} \sum_{\alpha, \alpha^{\prime}} \hat{\mathbf{R}}_{\beta \alpha} D_{\alpha \alpha^{\prime}} \hat{\mathbf{R}}_{\alpha^{\prime} \beta}^{*} .
$$

Here $\hat{\mathbf{R}}$ is the unitary transformation composed of all $A$-qubit rotations prior to the measurement:

$$
\hat{\mathbf{R}}_{\alpha \beta}=\bigotimes_{l=1}^{m} \hat{R}_{\alpha_{l} \beta_{l}}^{(l) *} .
$$

Only those subhistories that correspond to different $\beta$ are guaranteed to have 0 off-diagonal matrix element between them, and hence no quantum interference. However, different subhistories for a given $\beta$ preserve coherence and do not satisfy the consistency postulate of $\mathrm{CH}$ formalism. This makes our framework more general. Note also that we have not made any assumption about the time sequence of premeasurement 


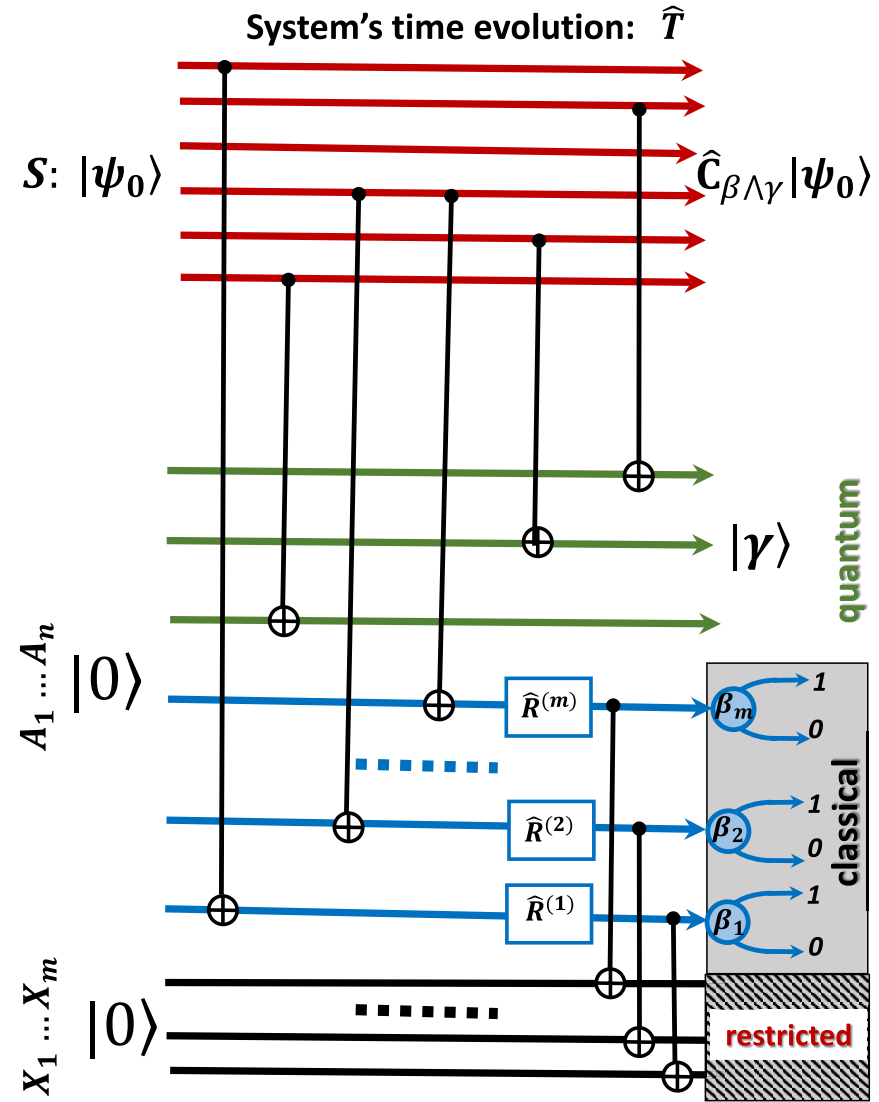

FIG. 4. Schematic representation of quantum history construction, with MMS-based branching of classical realities.

events that correspond to vectors $\beta$ and $\gamma$. In other words, our construction allows us to treat any information about the system's history in the same way, regardless of whether it corresponds to its past, its future, or any intermediate time. The term "branching" that we use here is often associated with Everett's multiworld interpretation of QM [4]. In our case, it refers to the emergence of distinct classical realities under the condition that $X$ qubits remain restricted (i.e., informationally isolated). Importantly, the branching in a general case occurs not in time, but rather as a function of the amount of information gained about the system.

For a given classical history $\beta$, we can determine the conditional probability of each subhistory $\beta \wedge \gamma$ that refines it: $p(\gamma \mid \beta)=\mathbf{D}_{\gamma \gamma}^{(\beta)} / \mathbf{T r}\left(\mathbf{D}_{\gamma \gamma^{\prime}}^{(\beta)}\right)$. If the outcome of a particular measurement $\gamma_{l}$ is not known, the probability of a given history is determined by taking the respective partial trace of the history density matrix, $\mathbf{T r}_{\gamma_{l}}\left(\mathbf{D}_{\gamma \gamma^{\prime}}^{(\beta)}\right)$, or, equivalently, by taking a sum of probabilities of all the compatible histories. Importantly, ignoring the result of a measurement, or not measuring a specific $A$ qubit, is not equivalent to not making that measurement on the system. As has been demonstrated in the previous section, the effect of any single premeasurement can be erased and quantum interference effects recovered. To do this, the rotation matrix for the corresponding $A$ qubit has to be given by the Hadamard transformation $\hat{R}^{(l)}=\hat{H}$ and the result $\beta_{l}=1$ has to be selected.

\section{SUMMARY}

In the present work, we revisited the long-standing quantum measurement problem and the issue of the quantumclassical divide. We worked within the conventional framework of QM but dropped the projection postulate or, equivalently, the wave-function collapse prescription. Instead, we formulated the minimalistic measurement scheme (MMS) that assumes that a part of a quantum system becomes restricted, i.e., unavailable for any future interactions. This procedure is a simplified version of the decoherence scheme that does not require coupling of the apparatus to an environment or an infinitely large supplementary subsystem. The classical domain emerges naturally, conditional upon the informational isolation of the restricted subsystem. Specifically, we used two groups on ancilla qubits: $A$ type and $X$ type. The former represents measurement apparatuses that eventually record classical information about the system, once the latter becomes restricted or lost. The entropy increase due to the informational isolation of $X$ qubits represents informational or thermodynamic cost of performing the measurement and extracting new information about the system. The probabilistic nature of QM is a direct consequence of this information loss during the measurement process.

There is an important duality of how the $X$ qubit can be viewed. On the one hand, it is simply a part of the extended isolated quantum system $(S+A+X)$. On the other hand, making $X$ restricted i.e. unavaliabe makes apparatus (A) classical. Conversely, if any information about $X$ qubits is recovered, the corresponding $A$ qubit cannot be treated as classical anymore (since $A$ and $X$ constitute a Bell pair, and nonclassical correlations could be observed). In other words, the boundary between quantum and classical domains is determined by the information that is being withheld or lost. The classicality is therefore both emergent and reversible property.

Due to the conscious choice of staying within the domain of conventional QM, our theory does not result in any experimental prediction that would differentiate it from the traditional Copenhagen QM. However, it opens an opportunity to observe the measurement process and emergence of classicality within a finite closed system, as well as reversing it upon revealing restricted $X$ qubits. Similar direct experimental verification of the full-scale EID scheme remains prohibitively hard.

In addition to conventional von Neumann-style projective measurements, MMS naturally describes the so-called generalized or weak quantum measurements (POVM type). Repeating this measurement multiple times, with different pairs of $A$ and $X$ qubits, results in a gradual wave-function collapse of the system, which can even be reversed, but with a diminishing probability. The basis in which the collapse would eventually occur is determined by the premeasurement process.

Building upon the MMS, we mapped a history of a quantum system onto a set of $A$ qubits. This construction resembles the consistent history $(\mathrm{CH})$ approach to quantum theory but is built entirely within the framework of conventional QM. In particular, the key element of $\mathrm{CH}$ formalism, the consistency postulate, is not automatically 
satisfied. Rather, different histories branch into different classical realities only upon the use of the MMS. Our framework deviates from $\mathrm{CH}$ in several ways. First, it is constructed within a conventional single-time Hilbert space; second, the chain operator that defines a given history may include nonprojective generalized measurements, and, finally, the quantum coherence is preserved for subhistories within each classically distinct history, i.e., they may violate the consistency postulate of $\mathrm{CH}$. When MMS is applied to the set of $A$ qubits, the branching of different classical realities occurs. Each of these realities corresponds to a particular generalized history operator. Importantly, our construction is nearly time-agnostic: while time ordering of certain premeasurement events is important, the subset of measured $A$ qubits (and the choice of basis for each of them) can be absolutely arbitrary.
The probabilistic nature of QM is one of its most heralded features. Einstein's quote, “God does not play dice with the universe," is commonly portrayed as one of his biggest blunders. However, as we have demonstrated, the stochastic outcome of the quantum measurements is a direct consequence of the information sacrifice required to perform any such measurement.

\section{ACKNOWLEDGMENTS}

The author thanks N. Tkachenko for inspiring this work, and A. Douglas, D. Lukin, M. Hybertson, and I. Zaliznyak for valuable discussions. This research was conducted in the Center for Functional Nanomaterials, which is a U.S. DOE Office of Science User Facility, at Brookhaven National Laboratory under Contract No. DE-SC0012704.
[1] J. von Neumann, Mathematical Foundation of Quantum Mechanics (Princeton University Press, Princeton, USA, 1955).

[2] P. A. M. Dirac, The Principles of Quantum Mechanics (Clarendon Press, Oxford, 1930).

[3] D. Bohm, Phys. Rev. 85, 166 (1952).

[4] H. Everett, Rev. Mod. Phys. 29, 454 (1957).

[5] J. A. Wheeler, Rev. Mod. Phys. 29, 463 (1957).

[6] E. P. Wigner, Am. J. Phys. 31, 6 (1963).

[7] S. Saunders, J. Barrett, A. Kent, and D. Wallace, Many Worlds?: Everett, Quantum Theory, and Reality (Oxford University Press, Oxford, 2010).

[8] W. H. Zurek, Rev. Mod. Phys. 75, 715 (2003).

[9] W. H. Zurek, Phys. Today 44(10), 36 (1991).

[10] M. Gell-Mann and J. B. Hartle, Phys. Rev. D 47, 3345 (1993).

[11] M. Schlosshauer, Rev. Mod. Phys. 76, 1267 (2004).

[12] N. J. Cerf and C. Adami, Phys. Rev. Lett. 79, 5194 (1997).

[13] N. J. Cerf and C. Adami, Physica D 120, 62 (1998).

[14] K. Modi, A. Brodutch, H. Cable, T. Paterek, and V. Vedral, Rev. Mod. Phys. 84, 1655 (2012).

[15] C. H. Bennett and D. P. DiVincenzo, Nature (London) 404, 247 (2000).

[16] L. del Rio, J. Aberg, R. Renner, O. Dahlsten, and V. Vedral, Nature (London) 474, 61 (2011).

[17] J. M. R. Parrondo, J. M. Horowitz, and T. Sagawa, Nat. Phys. 11, 131 (2015).

[18] R. Steinigeweg, A. Khodja, H. Niemeyer, C. Gogolin, and J. Gemmer, Phys. Rev. Lett. 112, 130403 (2014).
[19] S. Goldstein, J. L. Lebowitz, R. Tumulka, and N. Zanghì, Phys. Rev. Lett. 96, 050403 (2006).

[20] R. Landauer, IBM J. Res. Dev. 5, 183 (1961).

[21] C. H. Bennett, Stud. Hist. Phil. Mod. Phys. B 34, 501 (2003).

[22] J. S. Bell, Physics (Long Island City, N. Y.) 1, 195 (1964).

[23] J. S. Bell, Rev. Mod. Phys. 38, 447 (1966).

[24] K. Kraus, States, Effects and Operations: Fundamental Notions of Quantum Theory (Springer Verlag, 1983).

[25] M. Nielsen and I. Chuang, Quantum Computation and Quantum Information (Cambridge University Press, 2000).

[26] A. Peres, Quantum Theory: Concepts and Methods (Kluwer Academic Publishers, 1993).

[27] Y. H. Kim, R. Yu, S. P. Kulik, Y. Shih, and M. O. Scully, Phys. Rev. Lett. 84, 1 (2000).

[28] S. P. Walborn, M. O. Terra Cunha, S. Pádua, and C. H. Monken, Phys. Rev. A 65, 033818 (2002).

[29] A. N. Korotkov and A. N. Jordan, Phys. Rev. Lett. 97, 166805 (2006).

[30] N. Katz, M. Neeley, M. Ansmann, R. C. Bialczak, M. Hofheinz, E. Lucero, A. O'Connell, H. Wang, A. N. Cleland, J. M. Martinis, and A. N. Korotkov, Phys. Rev. Lett. 101, 200401 (2008).

[31] R. B. Griffiths, J. Stat. Phys. 36, 219 (1984).

[32] C. J. Isham, J. Math. Phys. 35, 2157 (1994).

[33] R. Omnes, Rev. Mod. Phys. 64, 339 (1992).

[34] H. F. Dowker and J. J. Halliwell, Phys. Rev. D 46, 1580 (1992). 